\title{
Effect and Mechanism of Acid-Induced Soy Protein Isolate Gels as Influenced by Cellulose Nanocrystals and Microcrystalline Cellulose
}

\author{
Xueqi Jin ${ }^{1}$, Ruijing $\mathrm{Qu}{ }^{1}$, Yong Wang ${ }^{2}{ }^{\oplus}$, Dong $\mathrm{Li}^{3}$ and Lijun Wang ${ }^{1, *}$ \\ 1 Beijing Key Laboratory of Functional Food from Plant Resources, College of Food Science and Nutritional \\ Engineering, China Agricultural University, P.O. Box 50, 17 Qinghua Donglu, Beijing 100083, China; \\ 13935285477@163.com (X.J.); qrj@cau.edu.cn (R.Q.) \\ 2 School of Chemical Engineering, The University of New South Wales, Sydney, NSW 2052, Australia; \\ benjaminwy@gmail.com \\ 3 Beijing Advanced Innovation Center for Food Nutrition and Human Health, National Energy R \& D Center \\ for Non-Food Biomass, College of Engineering, China Agricultural University, Beijing 100083, China; \\ dongli@cau.edu.cn \\ * Correspondence: wlj@cau.edu.cn
}

check for updates

Citation: Jin, X.; Qu, R.; Wang, Y.; Li, D.; Wang, L. Effect and Mechanism of Acid-Induced Soy Protein Isolate Gels as Influenced by Cellulose Nanocrystals and Microcrystalline Cellulose. Foods 2022, 11, 461. https://doi.org/10.3390/foods 11030461

Academic Editor: Soottawat Benjakul

Received: 15 January 2022 Accepted: 31 January 2022

Published: 3 February 2022

Publisher's Note: MDPI stays neutral with regard to jurisdictional claims in published maps and institutional affiliations.

Copyright: (C) 2022 by the authors. Licensee MDPI, Basel, Switzerland. This article is an open access article distributed under the terms and conditions of the Creative Commons Attribution (CC BY) license (https:// creativecommons.org/licenses/by/ $4.0 /)$.

\begin{abstract}
The effects of cellulose nanocrystals (CNC) and microcrystalline cellulose (MCC) on the gel properties and microstructure of glucono- $\delta$-lactone-induced soy protein isolate (SPI) gels were investigated. The water-holding capacity, gel strength, and viscoelastic modulus of CNC-SPI gels were positively associated with CNC concentration from 0 to $0.75 \%(w / v)$. In contrast, MCC-SPI gels exhibited decreased water-holding capacity, gel strength, and viscoelastic modulus. All composite gels displayed high frequency dependence and the typical type I (strain thinning) network behavior. Changes in viscoelasticity under large strain were correlated with differences in the microstructure of SPI composite gels. Scanning electron microscopy (SEM) and confocal laser scanning microscopy (CLSM) showed that CNC were more evenly and steadily distributed in the protein matrix and formed a compact network structure. In contrast, MCC-SPI gels exhibited a discontinued and rough gel network with some large aggregates and pores, in which MCC was randomly entrapped. Fourier transform infrared spectroscopy (FTIR) and molecular forces results revealed that no new chemical bonds were formed in the gelation process and that the disulfide bond was of crucial importance in the gel system. With the addition of CNC, electrostatic interactions, hydrophobic interactions, and hydrogen bonds in the SPI gel network were significantly strengthened. However, the incorporation of MCC might obstruct the connection of the protein network. It is concluded that both cellulose type and concentration affect gelling properties.
\end{abstract}

Keywords: CNC; MCC; acid-induced soy protein isolate gelation; rheology; microstructure

\section{Introduction}

Soy protein isolate (SPI), which mainly contains globulins glycinin (11S) and $\beta$ conglycinin (7S), has been extensively used in the food field due to its high nutritive values and functional properties [1]. In addition, as a plant-derived protein, SPI has attracted widespread attention due to its low cost, low pollution, low toxicity, and renewable property compared with animal-based proteins [2,3]. The formation of gel is regarded as one of the most important functions. Acid-induced soy protein gel can be achieved by preheating the protein solution to aggregates and then lowering the $\mathrm{pH}$ toward the isoelectric point by adding glucono- $\delta$-lactone (GDL) to reduce intermolecular repulsion [4]. However, pure protein gels are sensitive to environmental conditions and have poor stability, which significantly limit their application [5]. 
It has been demonstrated that polysaccharides can alter the functional properties of protein gels, resulting in the formation of resultant composite gels with different microstructural, mechanical, and sensory properties [6]. The protein-polysaccharide interactions can be either adverse or beneficial to the formation of gels depending on their nature, addition amount, and ratio as well as the gel conditions [7]. Thus, it is vital to systematically investigate protein-polysaccharide interactions in order to design and develop the desired texture and expand the applications of gels.

Cellulose nanocrystals (CNC), one of the nanocelluloses with rod structure, are typically obtained by sulfuric acid hydrolysis and have attracted extensive attention due to their unique physicochemical characteristics [8]. The high aspect ratio and Young's modulus, together with large specific surface area, richness in hydroxyl groups, negligible toxicity, and excellent biodegradability and biocompatibility, offer extensive application prospects for $\mathrm{CNC}$ in many fields [9]. Currently, the potential applications of CNC in the food industry have been deeply studied, such as to stabilize the emulsion, act as thickeners and flavor carriers, modify the digestion protein, and improve food packaging [10-12]. Microcrystalline cellulose (MCC), an insoluble hydrophilic cellulose derivate, is a white crystalline powder that has been approved for use as food additive [13]. It is used as a stabilizer, sorbent, and filler in food products and as a potential fat replacer [14,15].

In recent years, several studies have shown that insoluble cellulose significantly affects protein gelation. Zhuang et al. [16] demonstrated that sugarcane dietary fiber (420 and $177 \mu \mathrm{m}$ ) was trapped within the myofibrillar protein gels and improved gel functionality by changing the water distribution. Xiao et al. [17] revealed that the addition of CNC remarkably improved heat-induced whey protein isolate gels and exhibited better WHC, thermal stability, and textural and rheological properties. They also contributed to forming a uniform and dense gel structure. However, Ahmadi et al. [14] reported that both microand nanocrystalline cellulose decreased the viscoelastic modulus of whey protein gels and led to inhomogeneous rough networks. Ullah et al. [18] found that nanosized dietary fibers $(370 \mathrm{~nm})$ were distributed into tofu gel more uniformly and steadily than microsized dietary fibers $(110 \mu \mathrm{m})$ but obstructed the gelling properties more obviously.

In previous studies, researchers have focused more on heat-induced protein gels. To our knowledge, the acid-induced gelation mechanism for SPI gels loaded with cellulose crystalline particles is not entirely understood. It will be very interesting to explore the characteristics of MCC- and CNC-loaded protein gels, which could act as delivery systems for bioactive compounds. Furthermore, cellulose crystals can act as functional ingredients due to their nutritional properties and health benefits [11]. In this study, the effect of different sizes (nanometer and micrometer) and concentrations of cellulose crystals on SPI gels were elucidated. Small amplitude shear tests (SAOS) and large amplitude shear tests (LAOS) were used to analyze the rheological properties. Moreover, the water-holding capacity and microstructure of acid-induced SPI gels, along with their molecular forces, were also researched to propose a potential forming mechanism that can be helpful in expanding the application of cellulose in the food industry.

\section{Materials and Methods}

\subsection{Materials}

SPI (SD-100) (protein content $\geq 90 \%$, dry basis, PI = 4.5) was supplied by Pine Agritech Limited (Shandong, China). CNC and MCC were both acquired from North Century Cellulose Material Co., Ltd. (Jiangsu, China) and prepared by sulfuric acid hydrolysis from cotton pulp. The zeta potential value of CNC was $-45.7 \pm 2.3 \mathrm{mV}$, and the length and diameter of CNC were 100-500 and 10-50 nm, respectively. The zeta potential value of MCC $(\sim 20 \mu \mathrm{m})$ was $-10.3 \pm 1.9 \mathrm{mV}$. GDL and fluorescent brightener 28 were obtained from Shanghai Macklin Biochemical Co., Ltd. (Shanghai, China). Rhodamine B was provided by Beijing Yinghai fine chemical industry (Beijing, China). All other chemicals used in this study were of analytical grade. 


\subsection{Preparation of Stock Solutions and Hydrogels}

The gel samples were prepared using the methods reported by Bi et al. and Yan et al. $[19,20]$ with a minor modification. Briefly, SPI power $(8 \%, w / v)$ was dispersed in deionized water and stirred for $4 \mathrm{~h}$ at room temperature. Then, the solution was stored overnight at $4{ }^{\circ} \mathrm{C}$ for complete hydration, after which it was subjected to heat treatment at $90^{\circ} \mathrm{C}$ for $30 \mathrm{~min}$ in a water bath, followed by rapid cooling in tap water. The $\mathrm{pH}$ of the SPI dispersion was adjusted to 7.0. Suspensions of CNC or MCC $(2 \%, w / v)$ were prepared by dispersing powders in deionized water at room temperature by stirring for $1 \mathrm{~h}$ and then keeping it at $4{ }^{\circ} \mathrm{C}$ overnight.

At room temperature, the SPI was mixed with CNC by magnetic stirring for $20 \mathrm{~min}$. All of the mixtures had the same final SPI concentration $(5 \%, w / v)$, and the concentrations of CNC were $0,0.25,0.5$, and $0.75 \%(w / v)$. After the addition of GDL $(2 \%, w / v)$, the dispersions were immediately transferred to a $40{ }^{\circ} \mathrm{C}$ water bath and then incubated for $30 \mathrm{~min}$ to form CNC-SPI mixed gels, followed by rapid cooling in an ice-water bath. MCCSPI mixed gels ( $5 \%$ SPI; $0,0.25,0.5$, and $0.75 \%$ MCC) were obtained following the same method. All gels were stored at $4{ }^{\circ} \mathrm{C}$ overnight before further analysis.

\subsection{Water-Holding Capacity (WHC) and Gel Strength}

The WHC of SPI gels was determined based on the methods reported by Campbell et al. [21] with some modifications. Gel samples of around $5 \mathrm{~g}$ were centrifuged at $7000 \times$ $g$ for $10 \mathrm{~min}$ at $4{ }^{\circ} \mathrm{C}$. The WHC (\%) was defined as the percentage of gel weight after centrifugation relative to its initial weight.

A texture analyzer (TA-XT plus, Stable Micro System Co., UK) equipped with a P/0.5R cylindrical probe was used for measuring the gel strength of SPI gel samples. First, $30 \mathrm{~mL}$ gel samples were formed in $50 \mathrm{~mL}$ texture cups. The gels were then equilibrated at room temperature for $30 \mathrm{~min}$ before analysis. The gels were penetrated to a depth of $5 \mathrm{~mm}$ at a speed of $1 \mathrm{~mm} / \mathrm{s}$, and the trigger force was $5 \mathrm{~g}$.

\subsection{Rheology Tests}

Rheology measurements were performed using an AR2000ex rheometer (TA, Crawley, UK) with an aluminum parallel plate geometry ( $40 \mathrm{~mm}$ diameter, $1000 \mu \mathrm{m}$ gap). The acidinduced SPI gels were formed in situ on a Peltier plate. The dispersions mixed with GDL were immediately transferred between the plates of the rheometer and then surrounded by dimethyl silicone oil to minimize water evaporation during measurements.

\subsubsection{Gel Formation}

The samples were rapidly heated to $40{ }^{\circ} \mathrm{C}$ and kept for $30 \mathrm{~min}$. During the dynamic oscillatory time sweep tests, the storage moduli $\left(\mathrm{G}^{\prime}\right)$ was monitored as a function of time at a frequency of $1 \mathrm{~Hz}$ and a strain of $0.5 \%$ (within linear viscoelastic region). A kinetics model was used to characterize the relationship between $\mathrm{G}^{\prime}$ and time [19].

$$
\mathrm{G}^{\prime}(\mathrm{t})=\mathrm{G}_{\infty}^{\prime}\left(1-\mathrm{e}^{-\mathrm{k}\left(\mathrm{t}-\mathrm{tg}_{\mathrm{g}}\right)}\right)
$$

where $G_{\infty}^{\prime}$ indicates the estimated maximum $G^{\prime}$ value of gel sample $(\mathrm{Pa}), \mathrm{k}$ stands for the gelation rate constant $\left(\mathrm{s}^{-1}\right)$, and $\mathrm{tg}_{\mathrm{g}}$ is the time when gelling starts (s).

\subsubsection{Frequency Sweep Test}

Once the gelling process was complete, the sample was rapidly cooled to $20{ }^{\circ} \mathrm{C}$ for 5 min to prevent $\mathrm{s}$ decline in $\mathrm{pH}$ values, after which it was subjected to a frequency sweep ranging from 0.1 to $10 \mathrm{~Hz}$ at a strain of $0.5 \%$. The storage and loss moduli ( $\mathrm{G}^{\prime}$ and $\left.\mathrm{G}^{\prime \prime}\right)$ were monitored and recorded. A power law model was used to characterize the frequency dependencies of $\mathrm{G}^{\prime}$ and $\mathrm{G}^{\prime \prime}$ [22].

$$
\mathrm{G}^{\prime}=\mathrm{K}^{\prime} \cdot \omega^{\mathrm{n} \prime}
$$




$$
\mathrm{G}^{\prime \prime}=\mathrm{K}^{\prime \prime} \cdot \omega^{\mathrm{n}^{\prime \prime}}
$$

where $\mathrm{K}^{\prime}$ and $\mathrm{K}^{\prime \prime}$ represent power law constants $\left(\mathrm{Pa} / \mathrm{s}^{\mathrm{n}}\right)$, and $\mathrm{n}^{\prime}$ and $\mathrm{n}^{\prime \prime}$ are frequency indices (dimensionless).

\subsubsection{Large Amplitude Oscillatory Shear (LAOS) Test}

Large amplitude oscillatory shear (LAOS) test was performed at $20^{\circ} \mathrm{C}$ with strain sweep. The strain applied ranged from 0.1 to $100 \%$ at $1 \mathrm{~Hz}$. Raw data of the oscillation waveform was collected, and the MITlaos program (MITlaos beta) was used to analyze Lissajous curves [23,24].

\subsection{Scanning Electron Microscope (SEM)}

The microstructure of SPI gels was observed using the methods reported by Xiao et al. [25] with a minor modification. Gel samples were cut into cubes $\left(2 \mathrm{~mm}^{3}\right)$, immersed in $2.5 \%$ glutaraldehyde at $4{ }^{\circ} \mathrm{C}$ for $6 \mathrm{~h}$, and rinsed with phosphate buffer $(0.1 \mathrm{~mol} / \mathrm{L}, \mathrm{pH} 7.0)$ three times, followed by dehydration using ethanol solutions with gradient concentrations $(30,50,70,80,90$, and 100\%). After freeze-drying, gels were coated with gold and observed by SEM (SU3500, HITACHI, Japan) at $10 \mathrm{kV}$. Images were taken at $3000 \times$ magnification.

\subsection{Confocal Laser Scanning Microscopic (CLSM)}

A Leica CLSM (TCS SP5 II, Leica Microsystems CMS GmbH, Mannheim, Germany) equipped with an inverted microscope (Leica DMI6000, Wetzlar, Germany) was used to investigate the microstructure of SPI gels according to a previous study [19]. Rhodamine $\mathrm{B}(0.1 \%, w / v)$ and fluorescent brightener $28(0.1 \%, w / v)$ were used for staining proteins and $\mathrm{CNC} / \mathrm{MCC}$, respectively [11]. Before gelation, samples were stained with fluorescent dyes in a volume ratio of 100:1:1 and mixed well. The mixture was added onto a concave slide and sealed, followed by gelling at $40{ }^{\circ} \mathrm{C}$ for $30 \mathrm{~min}$. Rhodamine $\mathrm{B}$ and fluorescent brightener 28 were excited at 543 and $405 \mathrm{~nm}$, respectively, and the emission wavelengths were collected at 580 and $441 \mathrm{~nm}$, respectively. Images were captured at $100 \times$ magnification with a scale of $1024 \times 1024$ pixels.

\subsection{Fourier Transform Infrared Spectroscopy (FTIR)}

FTIR spectra of CNC, MCC, and SPI gels were recorded using a PerkinElmer infrared spectrometer (Spectrum 100, Perkin-Elmer Co., Waltham, MA, USA). The freeze-dried sample (2 mg) was thoroughly milled with $\mathrm{KBr}$ power $(200 \mathrm{mg})$ and then compressed into a thin film. Measurements were performed at room temperature and subjected to 32 scans in the scanning range of $4000-400 \mathrm{~cm}^{-1}$ with a spectral resolution of $4 \mathrm{~cm}^{-1}$.

\subsection{Measurement of Gel Solubility}

In order to explore the molecular forces of SPI gel, CNC-SPI gels, and MCC-SPI gels, gel solubility in different solvents was determined according to a previous study with some modifications [26,27]. The solvents were as follows: deionized water (S1), $0.086 \mathrm{~mol} / \mathrm{L}$ Tris-0.09 mol/L glycine-4 mmol/L Na 2 EDTA (pH 8.0) (S2), S2 containing 0.5\% SDS (S3), S3 containing $8 \mathrm{~mol} / \mathrm{L}$ urea (S4), and S4 containing 2\% $\beta$-mercaptoethanol (S5). Briefly, $0.4 \mathrm{~g}$ gel sample was thoroughly mixed with $10 \mathrm{~mL}$ of each solvent on a Vortex-Genie 2 mixer (Scientific Industries, INC., New York, NY, USA) for $2 \mathrm{~min}$. Then, the mixtures were incubated at $25^{\circ} \mathrm{C}$ for $20 \mathrm{~min}$ in a shaking water bath followed by centrifugation at $5000 \times g$ for $15 \mathrm{~min}$. The concentration of protein in the supernatant was measured using the BSA standard. Protein solubility was expressed as the ratio of the supernatant protein content to total protein content.

\subsection{Statistical Analysis}

All experiments were carried out in at least triplicate and are represented as mean \pm standard deviation. Statistical analysis was conducted by SPSS software (SPSS 20.0; IBM SPSS Statistics, Chicago, IL, USA) and MATLAB software (MATLAB R2012a, Natick, MA, 
USA). Differences were considered statistically significant $(p<0.05)$ using the one-way analysis of variance (ANOVA) with Duncan's test.

\section{Results}

\subsection{WHC and Gel Strength}

Water-holding capacity (WHC) is one of the important parameters of gel systems. It is closely related to the interactions between water and other components in the gel as well as the network structure [22]. As shown in Figure 1A, WHC of SPI gels containing CNC and MCC showed a contrary trend with increasing cellulose concentration. The WHC values of the CNC-SPI gels increased significantly with increasing CNC concentration. Addition of MCC lower than $0.5 \%$ did not affect WHC significantly, while higher MCC concentration $(0.75 \%)$ caused obvious reduction in WHC of about $9 \%(p<0.05)$.
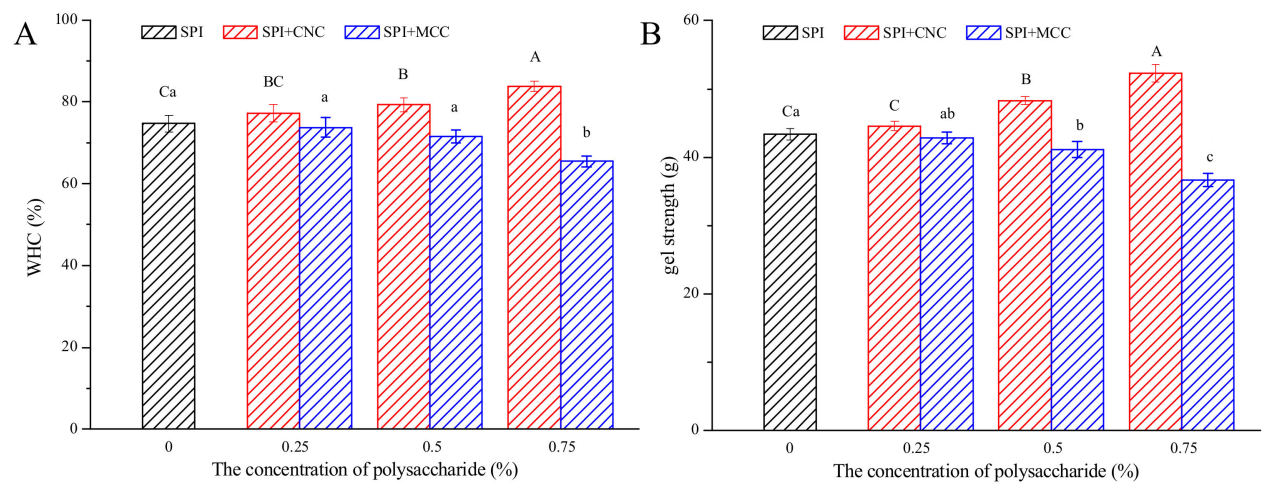

Figure 1. Water-holding capacity (A) and gel strength (B) of SPI gels containing different concentrations of CNC or MCC. Different upper case letters indicate significant differences among SPI gels containing different concentrations of CNC. Different lower case letters indicate significant differences among SPI gels containing different concentrations of MCC $(p<0.05)$.

In a composite gel system, water molecules may bind to functional groups of polysaccharides and proteins or get held in small meshes and pores of the gel network [28]. CNC are highly hydroxylated and contain abundant hydrophilic groups [29]. Hence, the increased number of hydrogen bonds might be one reason for the increase in WHC. Moreover, improvement in the WHC of CNC-SPI gels may be attributed to stronger intermolecular interaction in the gel system and the formation of a firm and stable network [25]. The results were in agreement with the study by Xiao et al. [17], who found that CNC could enhance WHC, gel strength, and thermal stability of heat-induced whey protein isolate gels and result in the formation of a well-structured gel network. However, MCC had an adverse effect on WHC of SPI gels. A similar trend was observed by López et al. [30], who found that sodium caseinate acid-induced gels containing espina corona gum presented lower WHC. The interaction between protein and MCC was limited due to its lower surface charge, which could make them more susceptible to rearrangements and induce the formation of locally denser clusters and larger pores [31]. Large pores have lower potency to immobilize water, allowing water to flow out easily under centrifugal forces [32]. Moreover, MCC tends to aggregate in high concentrations, which might obstruct protein-protein interactions. The formation of phase-separated structure might also have accounted for the decrease in WHC [33].

As shown in Figure 1B, incorporation of CNC and MCC significantly influenced gel strength. The results followed a similar trend to WHC, with CNC increasing the gel strength in a concentration-dependent manner and the addition of MCC decreasing the gel strength. It is generally accepted that gel strength is related to the arrangement of the protein network [17,22]. The incorporation of CNC and MCC might have affected the protein-protein interactions and protein aggregation, thus exhibiting different texture parameters. An increase in gel strength with increasing $\mathrm{CNC}$ concentration suggests the formation of a ho- 
mogeneous and cross-linked protein network. Insoluble large particles affect protein chain association, reducing protein-protein interaction [18]. Thus, the decrease in gel strength of MCC-SPI gels might be attributed to the decrease in protein-protein interaction and an unstable network structure. The results were consistent with the changes in interaction forces. In brief, the results of the WHC and gel strength prove that the addition of cellulose is an effective way to tune the characteristics of SPI gels.

\subsection{Rheological Tests}

\subsubsection{Gel Formation}

The elastic modulus $\left(\mathrm{G}^{\prime}\right)$ depends on the number of effective elastic junctions between strands [34]. Figure $2 \mathrm{~A}_{1}, \mathrm{~A}_{2}$ shows $\mathrm{G}^{\prime}$ development for CNC-SPI gels and MCC-SPI gels during their gelation processes, respectively. The tendency of $\mathrm{G}^{\prime}$ with time was similar. GDL hydrolyzed progressively, releasing protons to neutralize the negative charges on SPI as the $\mathrm{pH}$ value decreased. When the formation of SPI gel started, $\mathrm{G}^{\prime}$ increased sharply and then reached a maximum value. A similar gelation trend was observed by others [35].
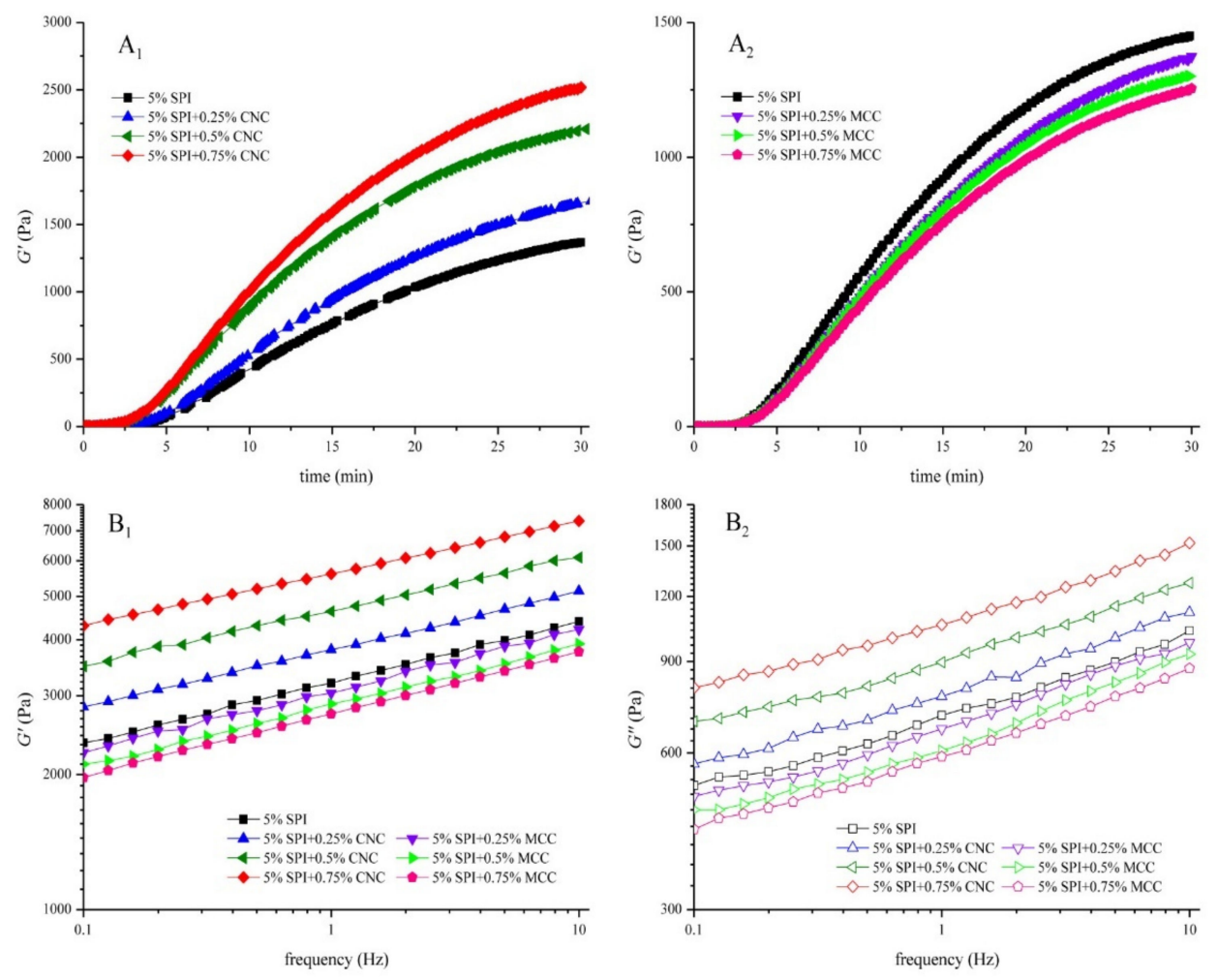

Figure 2. Linear rheology properties: time dependence of storage modulus $\mathrm{G}^{\prime}$ for $\mathrm{CNC}-\mathrm{SPI}$ gels $\left(\mathbf{A}_{\mathbf{1}}\right)$ and MCC-SPI gels $\left(\mathbf{A}_{\mathbf{2}}\right)$; changes in frequency dependence of storage modulus $\mathrm{G}^{\prime}\left(\mathbf{B}_{\mathbf{1}}\right)$ and loss modulus $G^{\prime \prime}\left(\mathbf{B}_{2}\right)$.

As summarized in Table 1, the kinetics model was used to fit the time sweep data using Equation (1) $\left(R^{2}>0.99\right)$. CNC exhibited noticeable increase in $\mathrm{G}^{\prime}$ of SPI gels, indicating a strong interaction between protein molecules and cellulose chains. $G_{\infty}^{\prime}$ increased and the gelation rate constant $\mathrm{k}$ and the gelation time $t_{\mathrm{g}}$ decreased significantly when the concentration of CNC increased $(p<0.05)$. The rod structure of CNC leads to great resistance [36]. The incorporation of $\mathrm{CNC}$ may strengthen the protein-protein interactions with increased relative density of protein aggregates, thus accelerating the early stage of gel formation [34,37]. Moreover, CNC could act as a thickening agent and might form hydrogen bonds with SPI [29]. 
Table 1. The parameter fitting results of the kinetics model for CNC-SPI gels and MCC-SPI gels.

\begin{tabular}{|c|c|c|c|c|}
\hline \multirow{2}{*}{$\begin{array}{c}\text { Sample } \\
(w / v)\end{array}$} & \multicolumn{3}{|c|}{$\mathbf{G}^{\prime}(\mathbf{t})=\mathbf{G}_{\infty}^{\prime}\left(1-\mathbf{e}^{-\mathbf{k}\left(\mathbf{t}-\mathbf{t}_{\mathrm{g}}\right)}\right)$} & \multirow{2}{*}{$R^{2}$} \\
\hline & $\mathrm{G}_{\infty}^{\prime}(\mathrm{Pa})$ & $\mathbf{k}\left(\mathrm{s}^{-1}\right)$ & $t_{g}(s)$ & \\
\hline $5 \%$ SPI & $2389 \pm 26^{\mathrm{Da}}$ & $0.00071 \pm 0.00001^{\mathrm{Aa}}$ & $213.93 \pm 5.50 \mathrm{Aa}$ & 0.995 \\
\hline $5 \%$ SPI $+0.25 \% \mathrm{CNC}$ & $2930 \pm 16^{C}$ & $0.00057 \pm 0.00005^{\mathrm{BC}}$ & $205.13 \pm 8.15^{\mathrm{A}}$ & 0.995 \\
\hline $5 \%$ SPI $+0.50 \% \mathrm{CNC}$ & $3470 \pm 65^{B}$ & $0.00065 \pm 0.00010^{\mathrm{AB}}$ & $135.43 \pm 21.06^{B}$ & 0.992 \\
\hline $5 \%$ SPI $+0.75 \% \mathrm{CNC}$ & $4598 \pm 47^{\mathrm{A}}$ & $0.00051 \pm 0.00008^{C}$ & $129.97 \pm 8.69^{\mathrm{B}}$ & 0.990 \\
\hline $5 \%$ SPI $+0.25 \% \mathrm{MCC}$ & $2193 \pm 10^{b}$ & $0.00065 \pm 0.00001^{b}$ & $203.70 \pm 8.01^{\mathrm{a}}$ & 0.995 \\
\hline $5 \%$ SPI $+0.50 \% \mathrm{MCC}$ & $2029 \pm 64^{c}$ & $0.00070 \pm 0.00003^{a}$ & $199.03 \pm 2.70^{\mathrm{a}}$ & 0.995 \\
\hline $5 \%$ SPI $+0.75 \%$ MCC & $2004 \pm 78^{c}$ & $0.00065 \pm 0.00003^{b}$ & $208.97 \pm 11.27^{\mathrm{a}}$ & 0.995 \\
\hline
\end{tabular}

Values are the mean values \pm standard deviation $(n=3)$. Different upper case letters superscripted indicate significant differences among SPI gels containing different concentrations of CNC $(p<0.05)$. Different lower case letters superscripted indicate significant differences among SPI gels containing different concentrations of MCC $(p<0.05)$.

However, in the case of MCC-SPI, $\mathrm{G}_{\infty}^{\prime}$ decreased while the gelation time $\mathrm{t}_{\mathrm{g}}$ showed no significant changes with increasing concentration of MCC $(p<0.05)$. $\mathrm{G}^{\prime}$ is closely related to the network structure and bond strength of the gel [38]. The incorporation of MCC might obstruct the connection of the protein network, thus reducing the number of effective strands and leading to a rougher network [18]. Similar findings were reported in other protein-polysaccharide gel systems [39].

\subsubsection{Frequency Dependence}

The frequency sweep plots of SPI gels are shown in Figures $2 \mathrm{~B}_{1}$ and $2 \mathrm{~B}_{2}$. The storage and loss moduli for all samples went up with the increase in frequency, which might be due to the acceleration of molecular mobility at high frequencies [40]. The strong frequency dependence indicates that the network was mainly formed by noncovalent physical crosslinks [41]. G' was far larger than $G^{\prime \prime}$, suggesting that all gels tended to be more elastic or solid-like. Consistent with the time sweep results, $G^{\prime}$ and $G^{\prime \prime}$ values of CNC-SPI gels increased with CNC concentration. In contrast, $\mathrm{G}^{\prime}$ and $\mathrm{G}^{\prime \prime}$ values showed a negative correlation with the concentration of MCC.

As shown in Table 2, the power law model fit well with the experimental data $\left(R^{2}>0.99\right)$. In the case of CNC-SPI gels, the values of $\mathrm{K}^{\prime}$ and $\mathrm{K}^{\prime \prime}$ increased significantly with increased CNC concentration $(p<0.05)$, indicating that the incorporation of CNC enhanced the viscoelasticity. The values of $n^{\prime}$ and $n^{\prime \prime}$ decreased after the addition of CNC $(p<0.05)$, suggesting that the sensitivity of the gels to the frequency was reduced. This might be due to the crystalline structure of $\mathrm{CNC}$ limiting polymer mobility [36]. The values of $K^{\prime}$ and $K^{\prime \prime}$ decreased significantly after the addition of MCC, while $\mathrm{n}^{\prime}$ increased and $\mathrm{n}^{\prime \prime}$ saw no significant difference $(p<0.05)$. This indicates that incorporation of MCC weakened the viscoelasticity of SPI gel, increased the elastic frequency dependence, and exerted no significant effect on the viscous frequency dependence. 
Table 2. The parameter fitting results of power law model for CNC-SPI gels and MCC-SPI gels.

\begin{tabular}{|c|c|c|c|c|c|c|}
\hline \multirow{2}{*}{$\begin{array}{c}\text { Sample } \\
(w / v)\end{array}$} & \multicolumn{3}{|c|}{$\mathrm{G}^{\prime}=\mathrm{K}^{\prime} \cdot \boldsymbol{\omega}^{\mathrm{n}^{\prime}}$} & \multicolumn{3}{|c|}{$G^{\prime \prime}=K^{\prime \prime} \cdot \omega^{n^{\prime \prime}}$} \\
\hline & $\mathbf{K}^{\prime}$ & $\mathbf{n}^{\prime}$ & $R^{2}$ & $\mathbf{K}^{\prime \prime}$ & $\mathrm{n}^{\prime \prime}$ & $R^{2}$ \\
\hline $5 \%$ SPI & $2541.7 \pm 39.2^{\mathrm{Da}}$ & $0.1339 \pm 0.0017^{\mathrm{Aa}}$ & 0.999 & $530.8 \pm 12.9 \mathrm{Da}$ & $0.1544 \pm 0.0011 \mathrm{Aa}$ & 0.995 \\
\hline $5 \%$ SPI $+0.25 \% \mathrm{CNC}$ & $3015.6 \pm 13.8^{C}$ & $0.1278 \pm 0.0008^{B}$ & 0.999 & $597.3 \pm 4.8^{\mathrm{C}}$ & $0.1487 \pm 0.0027^{\mathrm{A}}$ & 0.996 \\
\hline $5 \%$ SPI $+0.50 \% \mathrm{CNC}$ & $3604.5 \pm 51.5^{\mathrm{B}}$ & $0.1230 \pm 0.0004^{C}$ & 0.999 & $701.1 \pm 17.7^{\mathrm{B}}$ & $0.1382 \pm 0.0052^{\mathrm{B}}$ & 0.992 \\
\hline $5 \%$ SPI $+0.75 \% \mathrm{CNC}$ & $4515.0 \pm 28.2^{\mathrm{A}}$ & $0.1160 \pm 0.0002^{\mathrm{D}}$ & 0.999 & $820.3 \pm 9.6^{\mathrm{A}}$ & $0.1392 \pm 0.0009^{\mathrm{B}}$ & 0.994 \\
\hline $5 \%$ SPI $+0.25 \% \mathrm{MCC}$ & $2361.9 \pm 82.8^{b}$ & $0.1364 \pm 0.0010^{b}$ & 0.999 & $505.2 \pm 12.6^{\mathrm{a}}$ & $0.1545 \pm 0.0076^{\mathrm{a}}$ & 0.994 \\
\hline $5 \%$ SPI $+0.50 \% \mathrm{MCC}$ & $2197.9 \pm 46.3^{c}$ & $0.1367 \pm 0.0013^{b}$ & 0.999 & $467.4 \pm 11.6^{\mathrm{b}}$ & $0.1561 \pm 0.0057^{\mathrm{a}}$ & 0.996 \\
\hline $5 \%$ SPI $+0.75 \% \mathrm{MCC}$ & $2067.0 \pm 124.8^{c}$ & $0.1383 \pm 0.0013^{b}$ & 0.999 & $438.8 \pm 28.1^{\mathrm{b}}$ & $0.1562 \pm 0.0005^{\mathrm{a}}$ & 0.995 \\
\hline
\end{tabular}

Values are the mean values \pm standard deviation $(n=3)$. Different upper case letters superscripted indicate significant differences among SPI gels containing different concentrations of CNC $(p<0.05)$. Different lower case letters superscripted indicate significant differences among SPI gels containing different concentrations of MCC $(p<0.05)$.

\subsubsection{Nonlinear Rheology Properties}

The nonlinear rheology properties of gels reflect the sensory quality and mechanical properties related to food processing and contain valuable information about the microstructure [42]. The LAOS behaviors of SPI gels are shown in Figure $3 \mathrm{~A}_{1}, \mathrm{~A}_{2} \cdot \mathrm{G}^{\prime}$ and $\mathrm{G}^{\prime \prime}$ values of all gel samples remained constant regardless of strain amplitude at the linear viscoelastic region and then decreased dramatically with further increase in strain amplitude. This can be classified as type I gels (strain thinning). The creation rate parameter was negative $(a<0)$ and the decomposition rate parameter was positive $(b>0)$, indicating that the SPI gels were broken down easily and that the protein molecules had little chance to reestablish the network [24]. The number and types of protein-protein bonds, the aggregated size and shape during the "preaggregation" stage, and the gel network all influence the nonlinear viscoelastic behavior of gels [22,43]. Increasing CNC concentration increased both $\mathrm{G}^{\prime}$ and $\mathrm{G}^{\prime \prime}$, which might be due to the favorable interaction between $\mathrm{CNC}$ and protein molecules $[17,44]$. The values of $G^{\prime}$ and $G^{\prime \prime}$ decreased after the addition of MCC. It is noted that the decrease in viscoelastic moduli of MCC-SPI gels was slower than that of SPI gel under medium strain, which might be attributed to the energy dissipation caused by disruption of extensive agglomerates [45]. 

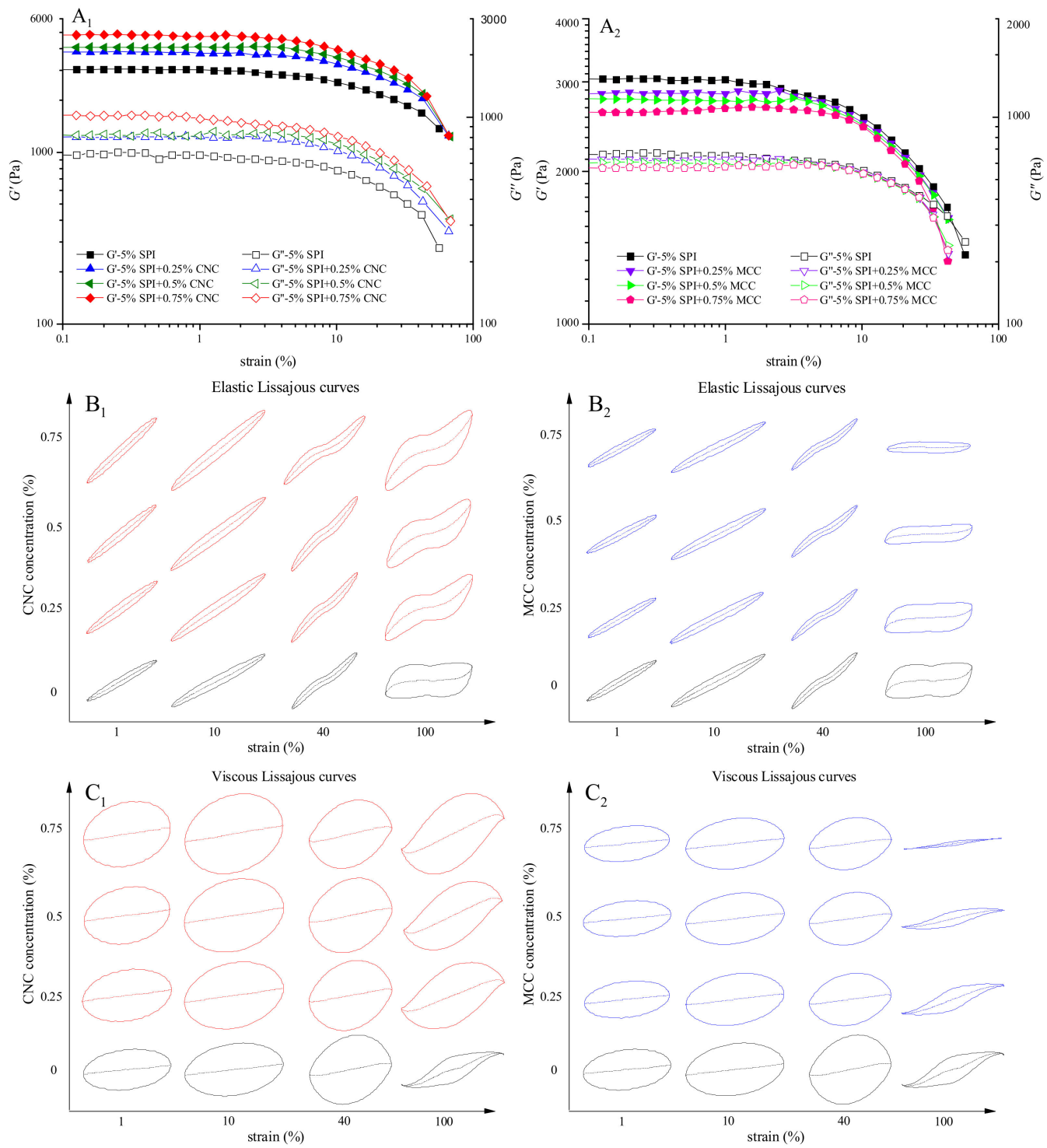

Figure 3. Nonlinear rheology properties: large amplitude oscillatory shear behavior of CNC-SPI gels $\left(\mathbf{A}_{1}\right)$ and MCC-SPI gels $\left(\mathbf{A}_{2}\right)$; elastic Lissajous curve of CNC-SPI gels $\left(\mathbf{B}_{1}\right)$ and MCC-SPI gels $\left(\mathbf{B}_{2}\right)$, and viscous Lissajous curves of CNC-SPI gels $\left(\mathbf{C}_{\mathbf{1}}\right)$ and MCC-SPI gels $\left(\mathbf{C}_{\mathbf{2}}\right)$. The black line is SPI gel, red line is CNC-SPI gels, and blue line is MCC-SPI gels in Lissajous curves. The coordinate axes of the same column in Lissajous curves are the same, and the solid and dashed lines represent the total stress and the decomposed stress, respectively.

Lissajous curves show the nonlinear behaviors of gels, with the shape and area of a Lissajous curve implying the viscoelastic response. The total stress is decomposed into elastic stress and viscous stress. The elastic Lissajous curves of SPI gels are depicted in Figure $3 B_{1}, B_{2}$, and the viscous Lissajous curves are presented in Figure $3 C_{1}, C_{2}$. As the systems entered the nonlinear region, the shape of the elastic Lissajous loops deformed from an ellipse to a rectangle and the strain rate curves changed from straight lines to distorted curves, indicating that the decrease in elasticity was faster than viscosity [46]. The area of the Lissajous loops expanded obviously with the addition of $\mathrm{CNC}$, demonstrating that the viscoelasticity of the gels increased [47]. This might be due to the strong resistance to permanent deformation by the formation of a well-structured gel network [48]. The incorporation of MCC might have formed a rougher gel network. The formation of fractures around the weakest junctions and existing pores would thus become more probable under 
large strain, which might account for the decrease in area of the Lissajous loops under large strain [49].

\subsection{Morphology and Microstructure}

\subsubsection{SEM}

The microstructures of SPI gels with or without different additions of CNC and MCC are presented in Figure 4. The control SPI gel showed a rough and disordered network with some large pores, indicating that the protein molecules were not well connected. The addition of CNC exhibited a smoother surface with more compact pores, facilitating the formation of homogeneous and dense networks. However, the gel network became disordered and fragmented with addition of MCC as evidenced by irregular aggregates. This might be responsible for the decreased WHC [31]. The large specific surface area of CNC was instrumental in binding the water molecules. The water might have migrated from the protein matrixes, resulting in "concentrated" SPI and thereby enhancing the gel network [16]. In contrast, MCC might have served as a passive filler that was not tightly embedded within the gel network, thus leading to decreased gel quality.

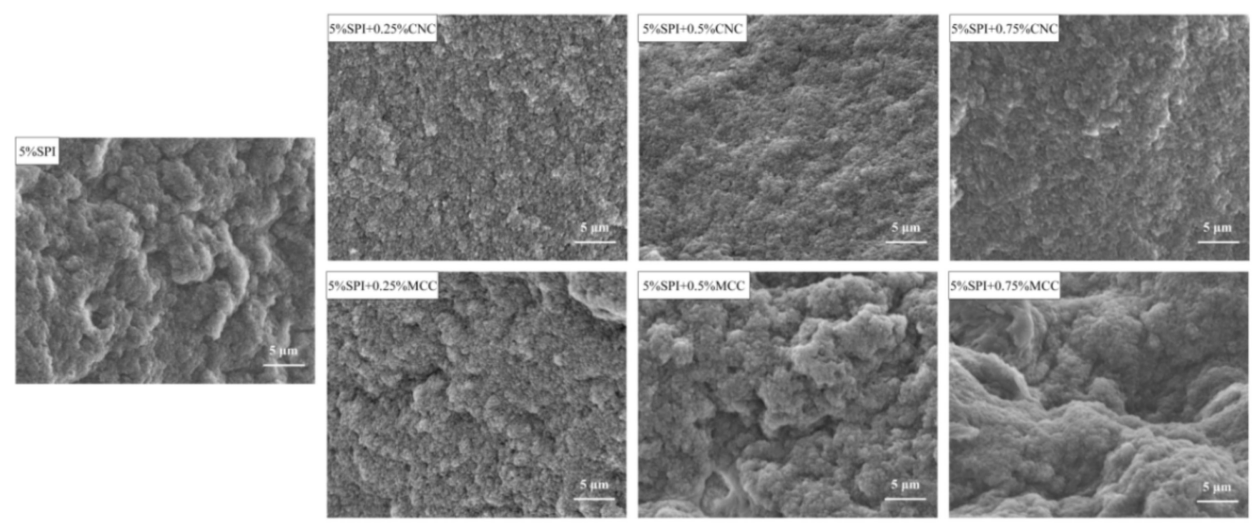

Figure 4. SEM images of SPI gels containing different concentrations of CNC or MCC.

\subsubsection{CLSM}

Figure 5 shows the CLSM images of SPI gel systems with or without CNC and MCC. Protein stained with rhodamine B appears as red, while CNC or MCC stained with fluorescent brightener 28 appear as white. The presence of CNC and MCC affected the distribution and size of the cavities. The pure SPI gel showed a heterogeneous network with different pore sizes. When CNC content increased, the protein continuous phase exhibited more compact and uniform structures, and the density of protein aggregates increased. The agglomerated CNC served as "active" fillers that were independently distributed in the gels [17]. However, MCC-SPI gels displayed inhomogeneous and loose microstructures with some large pores, where MCC was irregularly distributed in the gel matrix and tended to aggregate. Consistent with a previous study [18], CNC were distributed more uniformly into SPI gels compared with MCC, which might be due to the nanosize effect. The microstructure of protein-polysaccharide gel systems is determined by the competition between "gelation" and "phase separation" [4]. These CLSM images suggest that thermodynamic incompatibility occurred more dominantly between MCC and SPI, while "gelation" was more significant in CNC-SPI gels. 


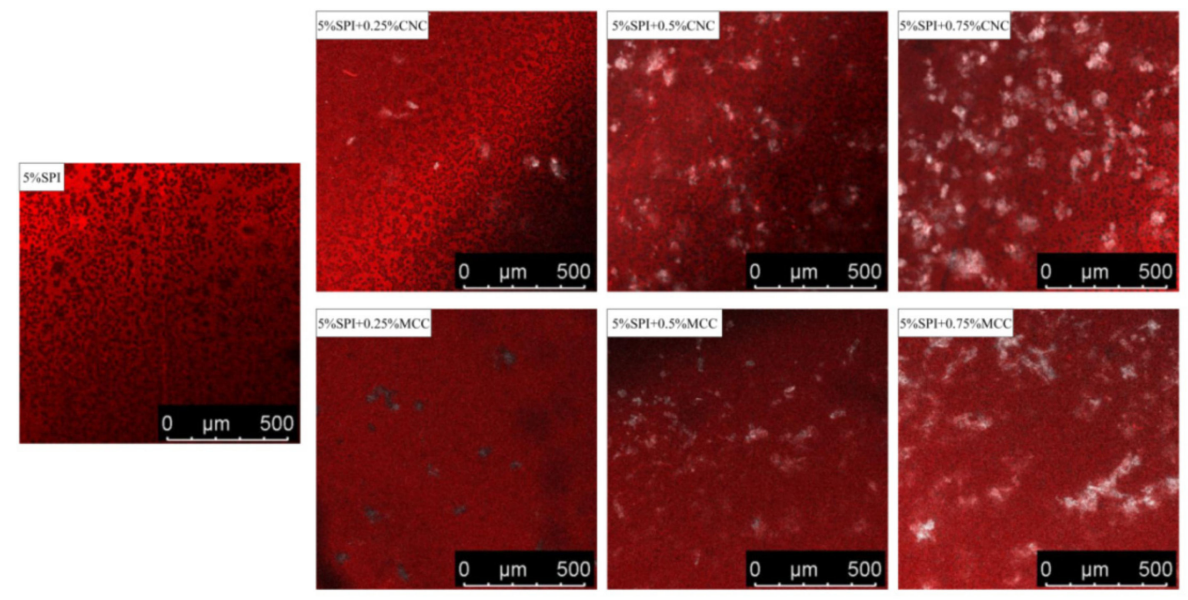

Figure 5. CLSM images of SPI gels containing different concentrations of CNC or MCC.

\subsection{Molecular Forces}

The FTIR spectra of CNC, MCC, and SPI gels are shown in Figure 6. The broad band at $3000-3700 \mathrm{~cm}^{-1}$ can be attributed to the $\mathrm{O}-\mathrm{H}$ and $\mathrm{N}-\mathrm{H}$ groups, reflecting more abundant hydroxyl groups on the surface of CNC compared to MCC [14]. This study found no clear changes in SPI gel spectra, indicating that no new chemical bonds formed in the gelation process. These results are consistent with previous studies showing physical entanglement and noncovalent interactions are the predominant driving forces between cellulose molecules and protein molecules [14,17]. The interaction mechanisms of the composite gels still need further investigated.


Figure 6. Molecular forces. FTIR spectra of CNC and SPI gels containing different concentrations of CNC ( $\left.\mathbf{A}_{\mathbf{1}}\right), \mathrm{MCC}$ and SPI gels containing different concentrations of MCC $\left(\mathbf{A}_{\mathbf{2}}\right)$, and chemical interaction forces of CNC-SPI gels $\left(\mathbf{B}_{1}\right)$ and MCC-SPI gels $\left(\mathbf{B}_{2}\right)$. Different letters indicate significant differences $(p<0.05)$. 
The protein solubility of SPI gels with or without CNC or MCC in five types of solvents were determined to explore the contribution of noncovalent and covalent bonds. The differences between two adjacent solvents represent electrostatic interaction, hydrophobic interaction, hydrogen bond, and disulfide bond [27]. As shown in Figure 6, hydrophobic interaction, hydrogen bond, and disulfide bond played a major role in the formation of SPI gels, where disulfide bond is of crucial importance. The number and accessibility of disulfide bonds are increased by heat treatment of SPI dispersion and GDL-induced gels pre-solutions [21]. Moreover, soluble SPI aggregates produced from high SPI concentration exhibit high contents of free sulfhydryl groups [35]. These might have accounted for the high disulfide bonds in the gels. Hydrophobic interaction contributed less in this study compared to a previous study [27], which might be due to the formation of larger aggregates after adjusting the $\mathrm{pH}$ with acid [49]. Furthermore, the unfolding of protein molecules with high SPI concentration was slightly hindered, and the exposed hydrophobic groups tended to re-encapsulated due to the association between thermal aggregates [35]. With the addition of $\mathrm{CNC}$, electrostatic interactions, hydrophobic interactions, and hydrogen bonds in SPI gel networks were significantly strengthened $(p<0.05)$, further verifying that CNC serve as a dehydrating agent to concentrated SPI solution. Moreover, the incorporation of $\mathrm{CNC}$ gives rise to more hydrogen bonds participating in the formation of the gel network. However, as the concentration of CNC increased to $0.75 \%$, disulfide bonds in SPI gel networks saw a weak decline $(p<0.05)$, which might be due to the steric effect of the self-agglomerated CNC [50]. Electrostatic attractive forces and hydrogen bonding existed between CNC and SPI, while the interaction between MCC and SPI was limited. For MCCSPI gels, hydrogen bonds and disulfide bonds were significantly decreased compared to pure SPI gel $(p<0.05)$. The incorporation of MCC might obstruct the connection of the protein network and have adverse effect on the accessibility of disulfide bonds, which further explains the decreased moduli and WHC [21,51].

\subsection{Schematic Mechanism}

Based on the above results, the potential mechanism for GDL-induced gelation of SPI with or without CNC or MCC is elucidated in Figure 7. The pure SPI gel exhibited a disordered and porous structure.

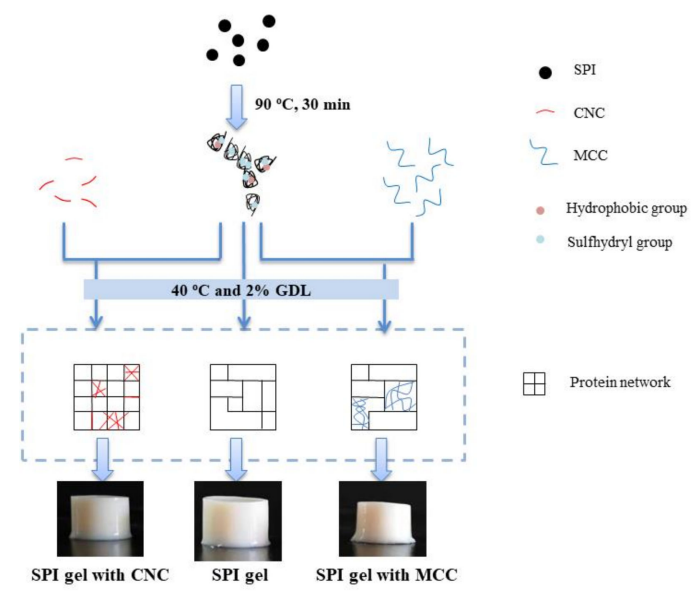

Figure 7. The suggested mechanism for the effects of CNC and MCC on acid-induced gelation of SPI.

The rod-shaped CNC have large specific surface area, good biocompatibility, and nanosize effect, which is highly hydroxylated and endowed with unique physicochemical properties. CNC might compete with proteins for water molecules and change the water distribution, leading to "concentrated" SPI and enhancing the gel network. The nanoscale CNC were more uniformly distributed in SPI gels, were steady, and tended to agglomerate. They might have acted as an active filler and contributed to the physical entanglement and hydrogen bonds. As the concentration of CNC increased, electrostatic interactions, 
hydrophobic interactions, and hydrogen bonds in the SPI gel network were strengthened, but higher $\mathrm{CNC}$ concentration $(0.75 \%)$ caused a weak reduction in disulfide bonds. In any case, the incorporation of CNC promoted the formation of a compact, regular, and firm GDL-induced SPI gel network with improvement in functional properties.

In contrast, the micrometer-scale MCC with fewer surface charges was irregularly and randomly distributed in SPI gels, which acted as a passive fill within the protein and influenced the gel network integrity and continuity. Moreover, after the incorporation of MCC, hydrogen bonds and disulfide bonds in the gels were significantly decreased. Thermodynamic incompatibility seemed to occur more dominantly and before protein gelation, which promoted the formation of microphase separation structures, resulting in rough, disordered, and uneven network structures that corresponded to low WHC, gel strength, and viscoelastic moduli.

\title{
4. Conclusions
}

In this study, the effects of addition of CNC and MCC, which could act as potential functional ingredients, on acid-induced SPI gels were investigated. The significant improvement in WHC, gel strength, and rheological properties of CNC-SPI gels indicate that the gel network structures were strengthened. Naturally sourced CNC can be used as a potential gel modifier, which plays a key role in forming CNC-SPI gels by changing the water distribution and constructing a more compact and homogeneous network structure. In contrast, the incorporation of MCC decreased WHC, gel strength, and $G^{\prime}$ and $G^{\prime \prime}$ value of gels and impeded the protein gelation process, resulting in the formation of a rougher network with some large aggregates and pores. Both the type and concentration of cellulose significantly affected the formation of acid-induced SPI gels. Unexpectedly, CNC and MCC had the opposite effect when introduced into SPI gels, with the strengthening effect of CNC proving much greater than the weakening effect of MCC. These findings provide a preliminary understanding of the interaction between protein molecules and cellulose chains, which can be applied to tune the characteristics of SPI gels, develop novel food products, and expand the potential application of cellulose in the food industry. Further work should focus on the competition between "phase separation" and "protein network formation" of composite gels and the design of delivery systems for bioactive compounds.

\begin{abstract}
Author Contributions: Conceptualization and methodology, X.J. and R.Q.; investigation and formal analysis, X.J.; resources, L.W. and D.L.; data curation, X.J.; writing—original draft preparation, X.J.; writing-review and editing, Y.W.; visualization, X.J.; supervision, L.W.; project administration, L.W.; funding acquisition, L.W. and D.L. All authors have read and agreed to the published version of the manuscript.
\end{abstract}

Funding: This research was funded by the National Natural Science Foundation of China (Project No. 31771896)

Data Availability Statement: Data is contained within the article.

Conflicts of Interest: The authors declare no conflict of interest.

\section{References}

1. Silva, J.; Cochereau, R.; Schmitt, C.; Nicolai, T.; Chassenieux, C. Heat-induced gelation of plant globulins. Curr. Opin. Food Sci. 2019, 27, 18-22. [CrossRef]

2. Abaee, A.; Mohammadian, M.; Jafari, S.M. Whey and soy protein-based hydrogels and nano-hydrogels as bioactive delivery systems. Trends Food Sci. Technol. 2017, 70, 69-81. [CrossRef]

3. Mohammadinejad, R.; Maleki, H.; Larrañeta, E.; Fajardo, A.R.; Nik, A.B.; Shavandi, A.; Sheikhi, A.; Ghorbanpour, M.; Farokhi, M.; Govindh, P.; et al. Status and future scope of plant-based green hydrogels in biomedical engineering. Appl. Mater. Today 2019, 16, 213-246. [CrossRef]

4. Chang, Y.; Li, D.; Wang, L.; Bi, C.; Adhikari, B. Effect of gums on the rheological characteristics and microstructure of acid-induced SPI-gum mixed gels. Carbohydr. Polym. 2014, 108, 183-191. [CrossRef]

5. Maltais, A.; Remondetto, G.E.; Subirade, M. Soy protein cold-set hydrogels as controlled delivery devices for nutraceutical compounds. Food Hydrocoll. 2009, 23, 1647-1653. [CrossRef] 
6. De Jong, S.; van de Velde, F. Charge density of polysaccharide controls microstructure and large deformation properties of mixed gels. Food Hydrocoll. 2007, 21, 1172-1187. [CrossRef]

7. Gaaloul, S.; Turgeon, S.L.; Corredig, M. Influence of shearing on the physical characteristics and rheological behaviour of an aqueous whey protein isolate-kappa-carrageenan mixture. Food Hydrocoll. 2009, 23, 1243-1252. [CrossRef]

8. Trache, D.; Hussin, M.H.; Haafiz, M.; Thakur, V.K. Recent progress in cellulose nanocrystals: Sources and production. Nanoscale 2017, 9, 1763-1786. [CrossRef]

9. Tang, J.; Sisler, J.; Grishkewich, N.; Tam, K.C. Functionalization of cellulose nanocrystals for advanced applications. J. Colloid Interface Sci. 2017, 494, 397-409. [CrossRef] [PubMed]

10. Huang, S.; Liu, X.; Chang, C.; Wang, Y. Recent developments and prospective food-related applications of cellulose nanocrystals: A review. Cellulose 2020, 27, 2991-3011. [CrossRef]

11. Liu, L.; Kong, F. Influence of nanocellulose on in vitro digestion of whey protein isolate. Carbohydr. Polym. 2019, 210, 399-411. [CrossRef] [PubMed]

12. $\mathrm{Mu}$, R.; Hong, X.; Ni, Y.; Li, Y.; Pang, J.; Wang, Q.; Xiao, J.; Zheng, Y. Recent trends and applications of cellulose nanocrystals in food industry. Trends Food Sci. Technol. 2019, 93, 136-144. [CrossRef]

13. Schuh, V.; Allard, K.; Herrmann, K.; Gibis, M.; Kohlus, R.; Weiss, J. Impact of carboxymethyl cellulose (CMC) and microcrystalline cellulose (MCC) on functional characteristics of emulsified sausages. Meat Sci. 2013, 93, 240-247. [CrossRef] [PubMed]

14. Ahmadi, M.; Madadlou, A.; Sabouri, A. Isolation of micro- and nano-crystalline cellulose particles and fabrication of crystalline particles-loaded whey protein cold-set gel. Food Chem. 2015, 174, 97-103. [CrossRef]

15. Gibis, M.; Schuh, V.; Weiss, J. Effects of carboxymethyl cellulose (CMC) and microcrystalline cellulose (MCC) as fat replacers on the microstructure and sensory characteristics of fried beef patties. Food Hydrocoll. 2015, 45, 236-246. [CrossRef]

16. Zhuang, X.; Zhang, W.; Liu, R.; Liu, Y.; Xing, L.; Han, M.; Kang, Z.; Xu, X.; Zhou, G. Improved gel functionality of myofibrillar proteins incorporation with sugarcane dietary fiber. Food Res. Int. 2017, 100, 586-594. [CrossRef] [PubMed]

17. Xiao, Y.; Liu, Y.; Wang, Y.; Jin, Y.; Guo, X.; Liu, Y.; Qi, X.; Lei, H.; Xu, H. Heat-induced whey protein isolate gels improved by cellulose nanocrystals: Gelling properties and microstructure. Carbohydr. Polym. 2020, 231, 115749. [CrossRef]

18. Ullah, I.; Hu, Y.; You, J.; Yin, T.; Xiong, S.; Din, Z.; Huang, Q.; Liu, R. Influence of okara dietary fiber with varying particle sizes on gelling properties, water state and microstructure of tofu gel. Food Hydrocoll. 2019, 89, 512-522. [CrossRef]

19. Bi, C.; Li, D.; Wang, L.; Adhikari, B. Effect of LBG on the gel properties of acid-induced SPI gels. LWT 2017, 75, 1-8. [CrossRef]

20. Yan, W.; Zhang, B.; Yadav, M.P.; Feng, L.; Yan, J.; Jia, X.; Yin, L. Corn fiber gum-soybean protein isolate double network hydrogel as oral delivery vehicles for thermosensitive bioactive compounds. Food Hydrocoll. 2020, 107, 105865. [CrossRef]

21. Campbell, L.J.; Gu, X.; Dewar, S.J.; Euston, S.R. Effects of heat treatment and glucono- $\delta$-lactone-induced acidification on characteristics of soy protein isolate. Food Hydrocoll. 2009, 23, 344-351. [CrossRef]

22. Zhao, H.; Chen, J.; Hemar, Y.; Cui, B. Improvement of the rheological and textural properties of calcium sulfate-induced soy protein isolate gels by the incorporation of different polysaccharides. Food Chem. 2020, 310, 125983. [CrossRef] [PubMed]

23. Ewoldt, R.H.; Winter, P.; Maxey, J.; McKinley, G.H. Large amplitude oscillatory shear of pseudoplastic and elastoviscoplastic materials. Rheol. Acta 2010, 49, 191-212. [CrossRef]

24. Hyun, K.; Wilhelm, M.; Klein, C.O.; Cho, K.S.; Nam, J.G.; Ahn, K.H.; Lee, S.J.; Ewoldt, R.H.; McKinley, G.H. A review of nonlinear oscillatory shear tests: Analysis and application of large amplitude oscillatory shear (LAOS). Prog. Polym. Sci. 2011, 36, 1697-1753. [CrossRef]

25. Xiao, Y.; Li, J.; Liu, Y.; Peng, F.; Wang, X.; Wang, C.; Li, M.; Xu, H. Gel properties and formation mechanism of soy protein isolate gels improved by wheat bran cellulose. Food Chem. 2020, 324, 126876. [CrossRef]

26. Jiang, L.; Ren, Y.; Xiao, Y.; Liu, S.; Zhang, J.; Yu, Q.; Chen, Y.; Xie, J. Effects of Mesona chinensis polysaccharide on the thermostability, gelling properties, and molecular forces of whey protein isolate gels. Carbohydr. Polym. 2020, 242, 116424. [CrossRef]

27. Wang, W.; Shen, M.; Jiang, L.; Song, Q.; Liu, S.; Xie, J. Influence of Mesona blumes polysaccharide on the gel properties and microstructure of acid-induced soy protein isolate gels. Food Chem. 2020, 313, 126125. [CrossRef]

28. Shen, Y.; Kuo, M.I. Effects of different carrageenan types on the rheological and water-holding properties of tofu. LWT 2017, 78, 122-128. [CrossRef]

29. Aziz, T.; Fan, H.; Zhang, X.; Haq, F.; Ullah, A.; Ullah, R.; Khan, F.U.; Iqbal, M. Advance study of cellulose nanocrystals properties and applications. J. Polym. Environ. 2020, 28, 1117-1128. [CrossRef]

30. López, D.N.; Galante, M.; Alvarez, E.M.; Risso, P.H.; Boeris, V. Effect of the espina corona gum on caseinate acid-induced gels LWT 2017, 85, 121-128. [CrossRef]

31. Zhang, S.; Hsieh, F.; Vardhanabhuti, B. Acid-induced gelation properties of heated whey protein-pectin soluble complex (Part I): Effect of initial pH. Food Hydrocoll. 2014, 36, 76-84. [CrossRef]

32. Roshanghias, S.; Madadlou, A. Functional and gel properties of whey protein nanofibrils as influenced by partial substitution with cellulose nanocrystal and alginate. Int. Dairy J. 2018, 81, 53-61. [CrossRef]

33. Huan, Y.; Zhang, S.; Vardhanabhuti, B. Effect of CMC molecular weight on acid-induced gelation of heated WPI-CMC soluble complex. J. Food Sci. 2016, 81, 502-507. [CrossRef] [PubMed]

34. Alting, A.C.; Weijers, M.; de Hoog, E.H.A.; van de Pijpekamp, A.M.; Cohen Stuart, M.A.; Hamer, R.J.; de Kruif, C.G.; Visschers, R.W. Acid-induced cold gelation of globular proteins: Effects of protein aggregate characteristics and disulfide bonding on rheological properties. J. Agric. Food Chem. 2004, 52, 623-631. [CrossRef] 
35. Wan, Y.; Li, Y.; Guo, S. Characteristics of soy protein isolate gel induced by glucono- $\delta$-lactone: Effects of the protein concentration during preheating. Food Hydrocoll. 2021, 113, 106525. [CrossRef]

36. Amini, E.N.; Tajvidi, M. Mechanical and thermal behavior of cellulose nanocrystals-incorporated Acrodur ${ }^{\circledR}$ sustainable hybrid composites for automotive applications. J. Compos. Mater. 2020, 54, 3159-3169. [CrossRef]

37. Kharlamova, A.; Chassenieux, C.; Nicolai, T. Acid-induced gelation of whey protein aggregates: Kinetics, gel structure and rheological properties. Food Hydrocoll. 2018, 81, 263-272. [CrossRef]

38. Liu, D.; Zhou, P.; Nicolai, T. Effect of Kappa carrageenan on acid-induced gelation of whey protein aggregates. Part I: Potentiometric titration, rheology and turbidity. Food Hydrocoll. 2020, 102, 105589. [CrossRef]

39. Matia-Merino, L.; Lau, K.; Dickinson, E. Effects of low-methoxyl amidated pectin and ionic calcium on rheology and microstructure of acid-induced sodium caseinate gels. Food Hydrocoll. 2004, 18, 271-281. [CrossRef]

40. Su, Y.; Dong, Y.; Niu, F.; Wang, C.; Liu, Y.; Yang, Y. Study on the gel properties and secondary structure of soybean protein isolate/egg white composite gels. Eur. Food Res. Technol. 2015, 240, 367-378. [CrossRef]

41. Anvari, M.; Joyner, H.S. Effect of formulation on structure-function relationships of concentrated emulsions: Rheological, tribological, and microstructural characterization. Food Hydrocoll. 2017, 72, 11-26. [CrossRef]

42. Melito, H.S.; Foegeding, C.R.D.A. Relating large amplitude oscillatory shear and food behavior: Correlation of nonlinear viscoelastic, rheological, sensory and oral processing behavior of whey protein isolate/ K-carrageenan gels. J. Food Process Eng. 2013, 36, 521-534. [CrossRef]

43. Ge, H.; Wu, Y.; Woshnak, L.L.; Mitmesser, S.H. Effects of hydrocolloids, acids and nutrients on gelatin network in gummies. Food Hydrocoll. 2021, 113, 106549. [CrossRef]

44. Tomczynska-Mleko, M.; Terpilowski, K.; Mleko, S. Physicochemical properties of cellulose/whey protein fibers as a potential material for active ingredients release. Food Hydrocoll. 2015, 49, 232-239. [CrossRef]

45. Xu, H.N.; Chu, C.; Wang, L.; Zhang, L. Droplet clustering in cyclodextrin-based emulsions mediated by methylcellulose. Soft Matter 2019, 15, 6842-6851. [CrossRef]

46. Bi, C.; Zhang, M.; Sun, D.; Hua, Z.; Zhu, Y.; Liu, Y.; Huang, Z.; Gao, F. A novel critical point for isotropic gel in rheological-fractal model. J. Food Eng. 2019, 244, 40-46. [CrossRef]

47. Qu, R.; Tang, M.; Wang, Y.; Li, D.; Wang, L. TEMPO-oxidized cellulose fibers from wheat straw: Effect of ultrasonic pretreatment and concentration on structure and rheological properties of suspensions. Carbohydr. Polym. 2021, 255, 117386. [CrossRef] [PubMed]

48. Anvari, M.; Joyner Melito, H.S. Effect of fish gelatin and gum arabic interactions on concentrated emulsion large amplitude oscillatory shear behavior and tribological properties. Food Hydrocoll. 2018, 79, 518-525. [CrossRef]

49. Homer, S.; Lundin, L.; Dunstan, D.E. Modifying the microstructure and mechanical properties of whey protein isolate gels using large deformation oscillatory strain. Food Hydrocoll. 2016, 61, 672-677. [CrossRef]

50. Li, K.; Zhong, Q. Aggregation and gelation properties of preheated whey protein and pectin mixtures at $\mathrm{pH} 1.0-4.0$. Food Hydrocoll. 2016, 60, 11-20. [CrossRef]

51. Rabiey, L.; Britten, M. Effect of protein composition on the rheological properties of acid-induced whey protein gels. Food Hydrocoll. 2009, 23, 973-979. [CrossRef] 